\title{
Crisis bifurcations in plane Poiseuille flow
}

\author{
Stefan Zammert and Bruno Eckhardt \\ Fachbereich Physik, Philipps-Universität Marburg, D-35032 Marburg, Germany \\ and J.M. Burgerscentrum, Delft University of Technology, 2628 CD Delft, The Netherlands
}

(Received 10 February 2015; published 30 April 2015)

\begin{abstract}
Many shear flows follow a route to turbulence that has striking similarities to bifurcation scenarios in lowdimensional dynamical systems. Among the bifurcations that appear, crisis bifurcations are important because they cause global transitions between open and closed attractors, or indicate drastic increases in the range of the state space that is covered by the dynamics. We here study exterior and interior crisis bifurcations in direct numerical simulations of transitional plane Poiseuille flow in a mirror-symmetric subspace. We trace the state space dynamics from the appearance of the first three-dimensional exact coherent structures to the transition from an attractor to a chaotic saddle in an exterior crisis. For intermediate Reynolds numbers, the attractor undergoes several interior crises, in which new states appear and intermittent behavior can be observed. The bifurcations contribute to increasing the complexity of the dynamics and to a more dense coverage of state space.
\end{abstract}

DOI: 10.1103/PhysRevE.91.041003

PACS number(s): 47.27.ed, 47.27.nd, 47.52.+j, 47.20.Ft

Numerical and experimental studies of pipe and plane Couette flow have demonstrated the significance of exact coherent structures and their bifurcations for the transition to turbulence [1-4]. Typically, these states appear in saddle-node bifurcations and then undergo further bifurcations. Initially, most of their complexity lies in the temporal dynamics, so that they are better characterized as chaotic rather than turbulent. With increasing Reynolds number, more temporal and spatial degrees of freedom are activated, until the complexity of a turbulent flow is established. Parallel to the increase in complexity comes a growth of the parts of state space that participate in the chaotic and turbulent dynamics. Studies of low-dimensional dynamical systems have revealed many routes to this increased complexity [5-8]. Several of them have already been discussed in the context of high-dimensional fluid systems, e.g., in the cases of plane Couette flow [2] or pipe flow [4,9]. One contribution of the present study is to document similar phenomenology in another canonical fluid system, plane Poiseuille flow (PPF). A second one is the demonstration of interior crisis and their contribution to increasing the complexity of the attractor and of the state space region covered by it.

PPF is the pressure driven flow between two parallel plates and differs from plane Couette flow and pipe flow because of the presence of a linear instability to transverse vortices, the so-called Tollmien-Schlichting modes [10-12]. It occurs at a critical Reynolds number of 5772.22 for a streamwise wave number $\alpha$ of 1.02056 (based on the center-line velocity and half the gap width), as determined by Orszag [13]. The bifurcation is subcritical, and reaches down to about $\operatorname{Re} \approx$ $2700[14,15]$ (for different wavelength). However, several experiments and numerical simulations show that turbulence occurs already at Reynolds numbers around 1000 [16-18], and hence well below the onset of Tollmien-Schlichting modes. Thus, the linear instability cannot explain the observed turbulence at low Reynolds numbers and the situation becomes analogous to that in plane Couette and pipe flow.

In order to determine the relevant saddle-node bifurcation in PPF we use the method of edge tracking, as described in [19] (see also [20]). The method traces the time evolution of initial conditions and uses bisection between an initial condition that returns to the laminar profile and one that becomes turbulent as an approximation to the one on the laminar-turbulent interface. In most cases the state evolves towards a simple attractor, such as a fixed point or a simple periodic orbit. It is then possible to continue the edge state in Reynolds number around the saddle-node bifurcation and to identify the upper branch solution. In recent work for plane Couette [2] and pipe flow [4] it was shown that the upper branch of the edge state undergoes various bifurcations resulting in a chaotic attractor. A boundary or exterior crisis ultimately destroys this stable attractor and creates the observed transient turbulence with its characteristic exponentially distributed lifetimes. The observed phenomenology is similar to what has been described and discussed in the context of chaotic dynamical systems $[7,8]$.

We will here show that this scenario is also present in plane Poiseuille flow and that another type of crisis bifurcation, the interior crisis, provides a mechanism by which the part of state space occupied by the chaotic attractor can increase. Furthermore, we will discuss mechanisms for the observed increase of lifetimes of chaotic transients [21].

For our numerical simulations we use the Channelfow code [22]. The Reynolds number $\operatorname{Re}=U_{0} d / \nu$ for the system is based on half the distance between the plates $d$, the maximum velocity of the laminar profile $U_{0}$, and the kinematic viscosity $v$. We take a coordinate system in which $x$ points in the streamwise, $z$ in the spanwise, and $y$ in the wall-normal direction. With the above choices for the dimensionless units the laminar profile becomes $\vec{u}_{l}(y)=[U(y), 0,0]$ with $U(y)=$ $1-y^{2}$. The total flow field $\vec{u}_{t}$ can be written as the sum of the laminar profile and a fluctuating component, $\vec{u}_{t}=\vec{u}_{l}+\vec{u}$. All simulations in this Rapid Communication are performed for constant mass flux and with no-slip boundary conditions at the walls. The calculations are restricted to a computational domain of length $2 \pi$, width $\pi$ (and height 2 ) in a subspace that is symmetric to reflections at the midplane and to spanwise reflections at the plane defined by $z=0$ :

$$
\begin{aligned}
& s_{y}:[u, v, w](x, y, z)=[u,-v, w](x,-y, z), \\
& s_{z}:[u, v, w](x, y, z)=[u, v,-w](x, y,-z) .
\end{aligned}
$$




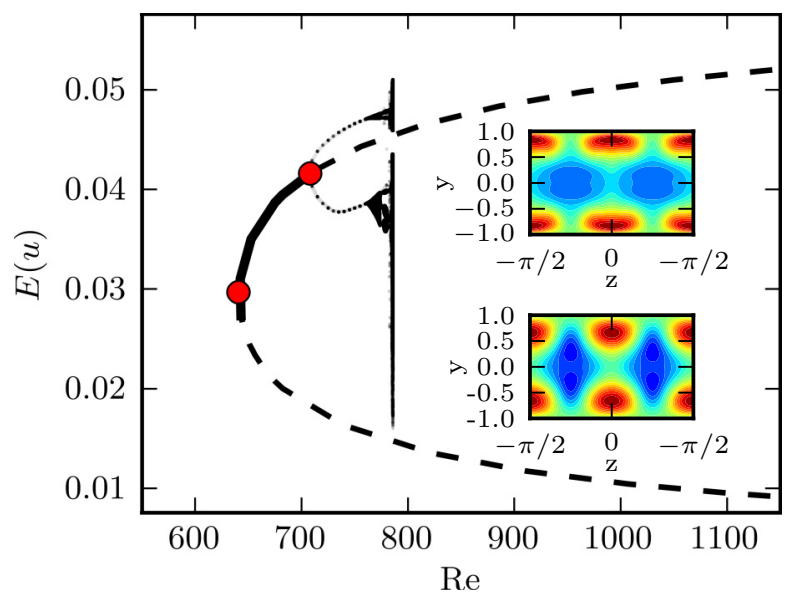

FIG. 1. (Color online) Bifurcation diagram for the traveling wave $T W_{E y z}$. Solid lines indicate a stable state, dashed lines an unstable state. Chaotic and periodic states are indicated by clouds of points obtained by plotting minima and maxima of their energy densities in the course of time. The red dots indicate the bifurcation points of $T W_{E y z}$ and $P O_{y z}$, respectively. The insets show the average streamwise velocity in the spanwise-wall-normal plane for the lower and the upper branches of $T W_{E y z}$ at $\mathrm{Re}=830$. The colors indicate low (blue) and high (red) velocity regions.

As in other studies [2,4], the restriction to a symmetric subspace stabilizes the exact coherent structures.

The numerical resolution is $N_{x} \times N_{y} \times N_{z}=48 \times 65 \times$ 48 modes. We checked higher values of $N_{x}$ and $N_{z}$ and found no significant changes in the bifurcations. The exact coherent structures and the location of the bifurcations vary with domain size, but the phenomenology is similar. The same applies to the localized coherent states and their bifurcations that appear in wider domains. Especially in large domains, the states become localized [23,24].

Using the technique of edge tracking it is possible to identify the edge state $[19,25]$ of this system. A trajectory on the laminar-turbulent boundary quickly reaches a state of constant energy. Since a stationary state is ruled out on account of the nonzero mean flow, the attractor in the laminar-turbulent boundary is a traveling wave. Indeed, a Newton search [26] for a relative equilibrium converges to a traveling wave, henceforth referred to as $T W_{E y z}$. The form of the state is indicated in the inset of Fig. 1. The traveling wave has the same symmetries as the mirror-symmetric traveling wave previously described by Nagata and Deguchi [27] and Gibson and Brandt [28].

We use a continuation method (see, e.g., [29]) to follow the solutions in Reynolds number around the saddle-node bifurcation at $\operatorname{Re}_{\mathrm{SN}}=641$. A stability analysis of the traveling wave shows that in the symmetry subspace the lower branch has one unstable eigenvalue and the upper branch is stable for $641<\operatorname{Re}<707$.

At $\operatorname{Re}=707$ the upper branch undergoes a Hopf bifurcation that creates a stable relative periodic orbit $P O_{y z}$. This orbit undergoes a Neimark-Sacker bifurcation at $\mathrm{Re}=761.5$ that creates a stable torus. In further bifurcations a chaotic attractor is generated. By plotting minima and maxima of the energy

$$
E(\vec{u})=\frac{1}{2 L_{x} L_{z}} \int_{0}^{L_{z}} \int_{-1}^{1} \int_{0}^{L_{x}} \vec{u}^{2} d x d y d z
$$

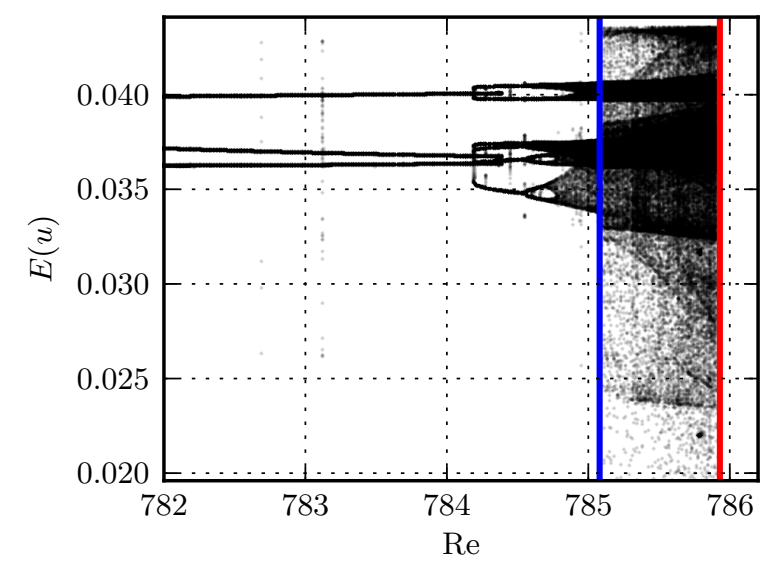

FIG. 2. (Color online) Magnification of the attractor in Fig. 1 in order to highlight the interior crisis (blue line) and the exterior crisis (red line). The states are visualized by plotting minima of $E(u)$ along trajectories.

of a trajectory on the attractor we are able to map out the bifurcation diagrams also in chaotic regions, as shown in Fig. 1. The mapping of the chaotic region becomes feasible due to the restriction by the shift-and-reflect symmetry that stabilizes the states. In the full system the entire bifurcation structure persists within an unstable subspace.

The magnification of the chaotic attractor in Fig. 2 highlights the two phenomena we want to focus on here: Slightly above $\operatorname{Re}=785$ (blue line) the size of the attractor expands and covers a larger fraction of the interval, and slightly below $\mathrm{Re}=786$ (red line) it disappears. Both changes are connected with crisis bifurcations [7,8]: an interior crisis in the first case, and an exterior crisis in the second case.

Slightly above $\mathrm{Re}=785$ the points on the attractor suddenly spread over a wider region, covering the area in state space with an energy $E$ between 0.023 and 0.044 . However, these parts of the state space are only visited occasionally, so that the points are less dense than in other parts. The reason for the sudden enlargement is a so-called interior crisis bifurcation [30,31], where a new state appears and new links to the attractor form. The appearance of the new states can be seen in the time series in Fig. 3. Just before the crisis, the range of the trajectories is limited to the interval $[0.033, \ldots, 0.042]$. Slightly above the crisis, excursions to lower values occur, with their number increasing with Re.

The type of transition can be determined from the distribution of times spent in the different regions. The state space region covered by the attractor before the crisis, referred to as phase $A$, contains trajectories that never drop below a threshold in energy, here taken to be $E_{t}=0.031$. If the trajectory drops below $E_{t}$, trajectories enter a different phase $B$, occupying a different region in state space. Indicators for phase $B$ are repeated excursions to values below $E_{t}$. Accordingly, if no excursions are noted for more than 250 time units, we conclude that the system has returned to phase $A$. With this prescription one can determine the distribution of times in phase $A$ as shown in Fig. 4(a). The plot contains data from trajectories with a total length of $5 \times 10^{6}$ time units. The data are shown semilogarithmically, so that the times are 


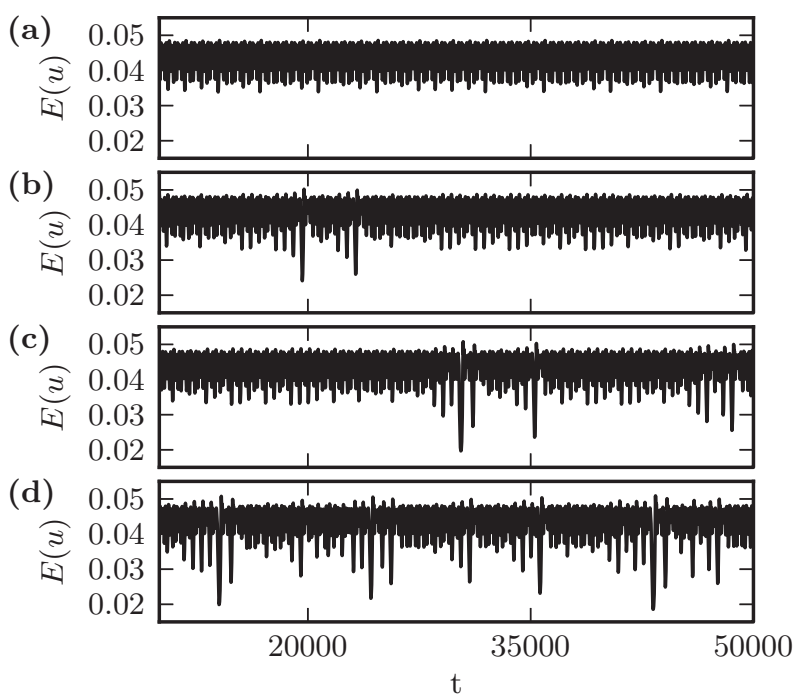

FIG. 3. Energy traces $E(t)$ for trajectories near the interior crisis. (a) $\operatorname{Re}=785.0$, slightly below the crisis. (b), (c), and (d) are for $\operatorname{Re}=785.46,785.75$, and 785.9, respectively, above the crisis. They show the characteristic intermittent bursts.
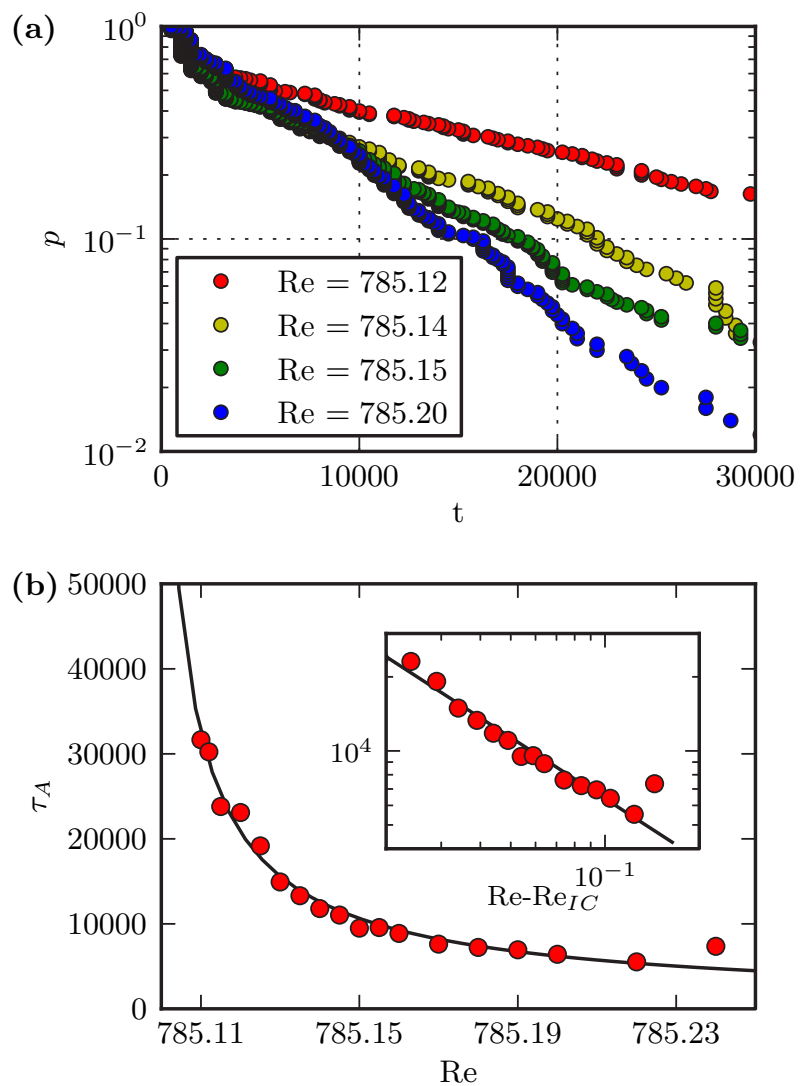

FIG. 4. (Color online) Intermittency near the interior crisis. (a) Probability to stay $t$ time units in the precrisis phase $A$. The times are exponentially distributed and the characteristic time scale $\tau_{A}$ increases with decreasing distance to $\mathrm{Re}_{\mathrm{IC}}$. (b) Variation of characteristic times with Reynolds number. The continuous line shows an algebraic fit to Eq. (4) with $\gamma=0.8$. The inset shows the data on a doubly logarithmic scale where on the abscissa the distance to the Reynolds number of the interior crisis is used. compatible with an exponential distribution, as expected for an interior crisis $[32,33]$. We then fit an exponential decay to the distribution to obtain the characteristic trapping times $\tau_{A}$ in phase $A$ and plot them versus Reynolds number in Fig. 4(b). Approaching the crisis point from above, the time in phase $A$ diverges since $B$ is never visited. According to $[8,32]$ the characteristic time varies as

$$
\tau_{A} \propto\left(\operatorname{Re}-\operatorname{Re}_{\mathrm{IC}}\right)^{-\gamma}
$$

with an exponent $\gamma$. We use $\operatorname{Re}_{\mathrm{IC}}=785.1$, as it is the lowest values of Re for which we observe excursions to phase $B$, and fit the the exponent to the data. In the present case we obtain a good fit to the data with $\gamma=0.8$.

The exponents for the interior crisis (as well as those of the exterior crisis) are expected to be larger or equal to $1 / 2$ for a smooth dynamical system [8,34]. For one-dimensional maps with a quadratic maximum they can be shown to be exactly $1 / 2$ but in higher dimensional systems the folding of the manifolds contributes to the dimensions, and higher exponents have been found $[7,8,35,36]$. Since the exponents depend on the eigenvalues at the point of bifurcation different crises can show different exponents, even in the same physical system.

Typical turbulent trajectories show an enormous temporal and spatial complexity that is difficult to create in a sequence of simple Neimark-Sacker or period-doubling bifurcations. As is evident form Fig. 3, the dynamics of the system is rather regular (but not periodic) before the interior crises and becomes increasingly more complicated (both in the range covered and in the complexity of the time signal) as the Reynolds number increases. Thus, the interior crisis bifurcation increases the complexity of the chaotic trajectories more dramatically than other local bifurcations and are an important contribution towards more turbulent time evolutions.

The second phenomenon we want to address here is the change in the dynamics near $\operatorname{Re}=786$, where the chaotic attractor suddenly disappears. Here, the attractor collides with the lower-branch state and turns into a chaotic saddle in a boundary or exterior crisis bifurcation [30]. It is a generic property of a chaotic saddle that the survival probabilities are exponentially distributed. To quantify this defining property of the boundary crisis, the survival probabilities for $\operatorname{Re}>\operatorname{Re}_{\mathrm{XC}}$ are calculated using the methods described by [37]. The survival probabilities are clearly exponential distributed with characteristic lifetimes that depend on the Reynolds number, as shown in Fig. 5. As in the case of the interior crisis they diverge as

$$
\tau \propto\left(\operatorname{Re}-\operatorname{Re}_{\mathrm{XC}}\right)^{-\delta}
$$

for Re near the Reynolds number $\mathrm{Re}_{\mathrm{XC}}$ of the crisis bifurcation. We fix $\operatorname{Re}_{\mathrm{XC}}=785.95$, since this is the lowest Reynolds number where we observed a trajectory that decays after showing transient chaotic dynamics for a long time. Best fits to the data are obtained for $\delta=1.5$, as expected for an exterior crisis. A dense sampling of initial conditions in the state space of the system combined with a fine scan of Reynolds numbers in the range between $\mathrm{Re}=778.3$ and $\mathrm{Re}=780.6$ reveals a small attractor $A_{1 a}$ inside of $A_{1}$. This attractor disappears at $\operatorname{Re}=780.6$ in another boundary crisis bifurcation and above 


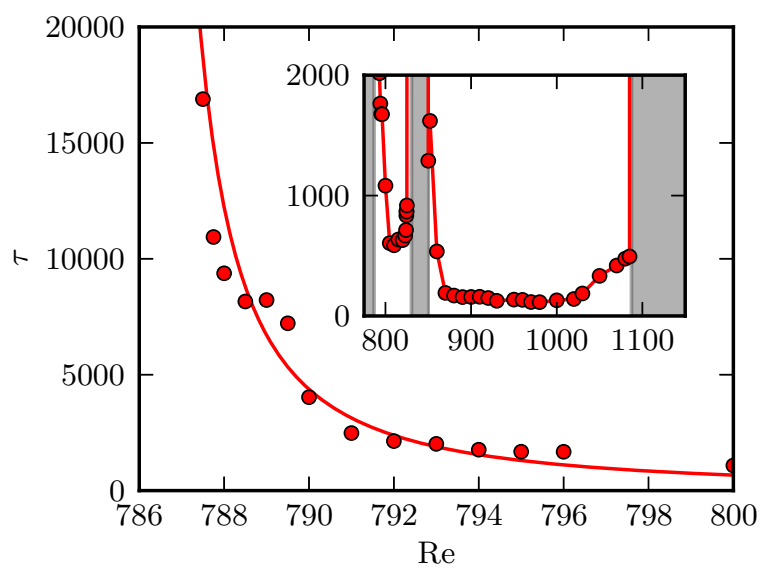

FIG. 5. (Color online) Characteristic lifetimes $\tau$ vs Reynolds number above the boundary crisis. The continuous line is an algebraic fit to Eq. (4) with $\delta=1.5$. The inset shows $\tau$ for a larger range in Re. The points are connected to guide the eye only. Regions where a stable attractor exists are shaded gray; the lifetime $\tau$ is infinite in these regions.

this Reynolds number initial conditions exist that transiently visit the saddle created by the boundary crisis of the attractor $A_{1 b}$ before suddenly settling down on $A_{1}$. An example for such a trajectory is shown in Fig. 6. Furthermore, no initial conditions can be found that transiently visit the saddle before becoming laminar. This behavior is strong evidence that $A_{1 a}$ lies completely inside of the basin of $A_{1}$. The presence of the chaotic saddle created in the boundary crisis of $A_{1 a}$ should lead to a second slope in the lifetime distribution as also seen in [36]. But since the basin of $A_{1 a}$ is very small compared to $A_{1}$ this slope does not influence the characteristic lifetimes in Fig. 5. The lifetimes for a larger range in Re are shown in the inset of Fig. 5. Following the boundary crisis the lifetimes of the chaotic transients first decrease, then start to increase again around $\operatorname{Re} \approx 815$, and diverge at $\operatorname{Re}=828$, where a second stable attractor $\left(A_{2}\right)$ appears. At slightly higher Re another attractor $\left(A_{3}\right)$ appears so that including the laminar state for a small range in $\mathrm{Re}$ the system has three attracting states.

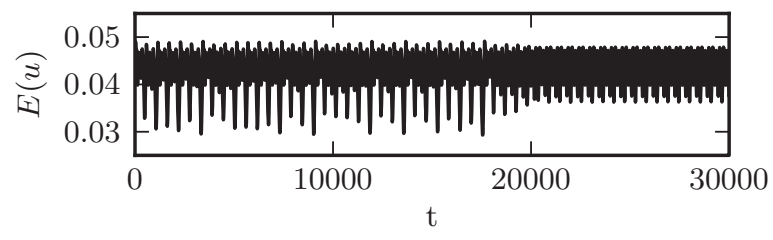

FIG. 6. Transition between saddle $A_{1 b}$ and attractor $A_{1}$ in the energy trace of a trajectory at $\operatorname{Re}=780.7$. The trajectory stays on the chaotic saddle $A_{1 b}$ for about 18000 time units before it suddenly switches to the stable attractor $A_{1}$.

$A_{2}$ and $A_{3}$ disappear in boundary crises at $\operatorname{Re}=837.5$ and $\mathrm{Re}=841.8$, respectively. After the boundary crisis of $A_{3}$ the lifetimes drop to even lower values than before the appearance of $A_{2}$. They decrease until $\operatorname{Re}=930$, where a lifetime of 126 is reached. Afterwards lifetimes increase again and eventually diverge at $\operatorname{Re}=1087$, where another attractor $A_{4}$ appears. The attractors $A_{2}-A_{4}$ appear in regions of the state space occupied by the large saddle created in the boundary crisis of $A_{1}$, as was checked using slices of the state space as in [36].

The crisis bifurcations analyzed here for PPF extend previous observations on Couette flow [36] and also pipe flow [38] in that they provide further examples of smaller chaotic saddles inside larger outer saddles and of bifurcations that contribute to an increase of the characteristic lifetimes and eventually to a more complex temporal dynamics. Moreover, the interior crises contribute to a more dense coverage of the state space of the system by the dynamics. Thereby, they pave the way for the transition to a chaotic saddle when the attractor collides with the saddle from the original saddlenode bifurcation. The connection to the phenomenology of low-dimensional dynamical systems and the appearance in a number of canonical flows suggests that this transition scenario is typical for the transition in shear flows.

We thank John Gibson for providing the Channelflow code and the participants and organizers of the EUROMECH Colloquium EC565 "Subcritical transition to turbulence" for fruitful discussions. This work was supported by the Deutsche Forschungsgemeinschaft within FOR 1182.
[1] B. Hof, C. W. H. van Doorne, J. Westerweel, F. T. M. Nieuwstadt, H. Faisst, B. Eckhardt, H. Wedin, R. R. Kerswell, and F. Waleffe, Science 305, 1594 (2004).

[2] T. Kreilos and B. Eckhardt, Chaos 22, 047505 (2012).

[3] G. Kawahara, M. Uhlmann, and L. van Veen, Annu. Rev. Fluid Mech. 44, 203 (2012).

[4] M. Avila, F. Mellibovsky, N. Roland, and B. Hof, Phys. Rev. Lett. 110, 224502 (2013).

[5] E. Ott, Chaos in Dynamical Systems (Cambridge University Press, Cambridge, UK, 2002).

[6] S. H. Strogatz, Nonlinear Dynamics and Chaos: With Applications to Physics, Biologiy, Chemistry and Engiennering (Perseus, Cambridge, MA, 1994).

[7] T. Tél and Y.-C. Lai, Phys. Rep. 460, 245 (2008).
[8] Y.-C. Lai and T. Tél, Transient Chaos-Complex Dynamics on Finite Time Scales (Springer, Berlin, 2011).

[9] F. Mellibovsky and B. Eckhardt, J. Fluid Mech. 709, 149 (2012).

[10] W. Heisenberg, Ann. Phys. 74, 577 (1924).

[11] C. C. Lin, On the development of turbulence, Ph.D. thesis, California Institute of Technology, Pasadena, 1944.

[12] L. Thomas, Phys. Rev. 91, 780 (1953).

[13] S. A. Orszag, J. Fluid Mech. 50, 689 (1971).

[14] J.-P. Zahn, J. Toomre, E. Spiegel, and D. Gough, J. Fluid Mech. 64, 319 (1974).

[15] I. Soibelman and D. I. Meiron, J. Fluid Mech. 229, 389 (1991).

[16] D. R. Carlson, S. E. Widnall, and M. F. Peeters, J. Fluid Mech. 121, 487 (1982).

[17] G. Lemoult, J.-L. Aider, and J. E. Wesfreid, Phys. Rev. E 85, 025303(R) (2012). 
[18] L. S. Tuckerman, T. Kreilos, H. Schrobsdorff, T. M. Schneider, and J. F. Gibson, Phys. Fluids 26, 114103 (2014).

[19] J. D. Skufca, J. A. Yorke, and B. Eckhardt, Phys. Rev. Lett. 96, 174101 (2006).

[20] S. Toh and T. Itano, J. Fluid Mech. 481, 67 (2003).

[21] B. Hof, J. Westerweel, T. M. Schneider, and B. Eckhardt, Nature (London) 443, 59 (2006).

[22] J. F. Gibson, Channelflow: A spectral Navier-Stokes simulator in $\mathrm{C}^{++}$, University of New Hampshire, Technical Report, 2012 (unpublished).

[23] K. Melnikov, T. Kreilos, and B. Eckhardt, Phys. Rev. E 89, 043008 (2014).

[24] S. Zammert and B. Eckhardt, J. Fluid Mech. 761, 348 (2014).

[25] T. M. Schneider, J. F. Gibson, M. Lagha, F. De Lillo, and B. Eckhardt, Phys. Rev. E 78, 037301 (2008).

[26] D. Viswanath, J. Fluid Mech. 580, 339 (2007).

[27] M. Nagata and K. Deguchi, J. Fluid Mech 735, R4 (2013).

[28] J. F. Gibson and E. Brand, J. Fluid Mech. 745, 25 (2014).

[29] H. Dijkstra, F. W. Wubs, A. K. Cliffe, E. Doedel, I. F. Dragomirescu, B. Eckhardt, A. Y. Gelfgat, A. L. Hazel, V.
Lucarini, A. G. Salinger, E. T. Phipps, J. Sanchez-Umbria, H. Schuttelaars, L. S. Tuckerman, and U. Thiele, Commun. Comput. Phys. 15, 1 (2014).

[30] C. Grebogi, E. Ott, and J. A. Yorke, Phys. Rev. Lett. 48, 1507 (1982).

[31] C. Grebogi, E. Ott, and J. A. Yorke, Phys. D Nonlinear Phenom. 7, 181 (1983).

[32] C. Grebogi, E. Ott, F. Romeiras, and J. A. Yorke, Phys. Rev. A 36, 5365 (1987).

[33] P. R. Muñoz, J. J. Barroso, A. C.-L. Chian, and E. L. Rempel, Chaos 22, 033120 (2012).

[34] C. Grebogi, E. Ott, and J. A. Yorke, Phys. Rev. Lett. 57, 1284 (1986).

[35] W. L. Ditto, S. Rauseo, R. Cawley, C. Grebogi, G.-H. Hsu, E. Kostelich, E. Ott, H. T. Savage, R. Segnan, M. L. Spano, and J. A. Yorke, Phys. Rev. Lett. 63, 923 (1989).

[36] T. Kreilos, B. Eckhardt, and T. M. Schneider, Phys. Rev. Lett. 112, 044503 (2014).

[37] M. Avila, A. P. Willis, and B. Hof, J. Fluid Mech. 646, 127 (2010).

[38] S. Altmeyer, A. Willis, and B. Hof, arXiv:1501.01989. 